\title{
Whole-Body Computed Tomography for Polytrauma Patients in the Emergency Department: Recommended or Unnecessary Radiation
}

\author{
Akram Bolbol*, Abdulhakam Ahmed, Osman EA Suliman and Abdullah Al-mater \\ Abduraboh
}

Surgical Department, King Fahad Hospital-Albaha, Saudi Arabia

*Corresponding author: Dr. Akram Bolbol, Surgical Department, King Fahad Hospital-Albaha, Saudi Arabia

\begin{abstract}
Background: Whole-body computed tomography (WBCT) is one of the standard non-invasive tests for trauma patients, to avoid the overuse of the WBCT and unnecessary radiation to the patients, a combination of evidence-based indications, approved guidelines and clinical decisions should be used.

This study was done to emphasize on limitation of unnecessary WBCT following international or local criteria along with clinical assessment and decision without compromising the patient's safety.

Methodology: This study was performed in King Fahad Hospital-Emergency Department in Albaha region in the kingdom of Saudi Arabia, the study was done in the period from January 2020 to December 2020, the study population is polytrauma patients of the age of 18 years old and above, it is a descriptive, retrospective database analysis of 233 patients diagnosed with polytrauma in the emergency department, all patients were received by emergency physicians and trauma team members, where they received their initial management and stabilized then sent to the Radiology department for WBCT according to the order by senior ED physician or on-call surgeon who is a member of the trauma team, Informed consent was taken according to the hospital protocol.
\end{abstract}

Results: Whole-body CT was carried out on 233 polytrauma patients arriving at the emergency department, the outcome of the WBCT was $n \approx 70(30 \%)$ were reported as $n \approx 87$ $(37.3 \%)$ they have a single organ involved in trauma and $n$ $\approx 76(32.7 \%)$ the reports came with multiple organs (more than 1) involved in trauma.

Conclusion: This study showed slight overuse of WBCT in our facility, $30 \%$ of the studies were reported.
The combination of the senior clinical staff application of appropriate local or international evidence-based criteria for performing WBCT will lead to avoiding a good number of unnecessary WBCT, radiation exposure, and side effects of contrast materials besides reducing the costs for the health services.

\section{Keywords}

Whole-body CT, Polytrauma, Single organ, Emergency department

\section{Introduction}

Whole-body CT is one of the standard non-invasive investigations in polytrauma patients in the emergency setting; proper clinical examination and standard indications for WBCT will reduce the unnecessary WBCT and convert it to the selected organ.

Discharging of patients from ED based on normal WBCT report is becoming questionable and controversial, furthermore, the high radiation dose of WBCT for younger patients and the risk of cancer cannot be ignored [1], one of the objectives of this article is to encourage health care systems and emergency settings to develop guidelines for utilization of WBCT in emergency departments [2].

Using other modalities of imaging in polytrauma patients like FAST and X-rays, might not be satisfactory for some physicians to avoid WBCT, this might be due to lack of skills of operating the bedside ultrasound machine, lack knowledge of interpretation the simple

Citation: Bolbol A, Ahmed A, Suliman OEA, Abduraboh AA (2022) Whole-Body Computed Tomography for Polytrauma Patients in the Emergency Department: Recommended or Unnecessary Radiation. Int J Surg Res Pract 9:136. doi.org/10.23937/2378-3397/1410136

Accepted: January 22, 2022; Published: January 24, 2022

Copyright: (C) 2022 Bolbol A, et al. This is an open-access article distributed under the terms of the Creative Commons Attribution License, which permits unrestricted use, distribution, and reproduction in any medium, provided the original author and source are credited. 
X-rays or for medicolegal purposes, although some researches showing that when medical staff using others modalities of imaging the result is reducing the percentage of negative WBCT [3].

Saudi Arabia has a high number of road trafficaccidents, 2020 according to the Ministry of health statistics there are total injuries of 25,561 and 4,618 deaths, the Saudi health system is providing the emergency departments and trauma centers with very advanced high technical machines for imaging and scanning of trauma patients in which we need to make a proper prioritization and utilization of it especially the CT machines to avoid the disadvantages of unnecessary radiation [4].

Many trauma centers have clear justifications for performing WBCT which is shared between the physicians and radiologists [5], however, there is several patients seem to be matching with the criteria for WBCT but the final report is normal WBCT, this safety margin of error looks acceptable for the most of the physicians and health system as well [4].

Whole-body CT enhanced with single contrast has special indications for detecting penetrating abdominopelvic trauma and suspected bowel injuries, studies are showing that single contrast is accurate as of the same of triple contrast (IV, Oral and rectal) [6], many physicians when they request a WBCT they are requesting contrast study either intravenous, oral or rectal as routine regardless the presents of abdominal trauma or penetrating injuries, contrast materials have many immediate or delayed side effects which should be considered by the treating physicians and to avoid it whenever it is possible [7].

It is the responsibility of the health system and quality sections in the hospital to provide the emergency system and physicians with established guidelines and protocols to reduce the overuse of WBCT in the polytrauma patient.

\section{Methods}

This study was performed in King Fahad HospitalEmergency Department in Albaha region in the kingdom of Saudi Arabia, the study was done in the period from January 2020 to December 2020, the study population is polytrauma patients of the age of 18 years and above, it is a descriptive, retrospective database analysis of 233 patients diagnosed with polytrauma in the emergency department, all patient was received by emergency physicians and trauma team members, they have received their initial management and stabilized then sent to the radiology department for WBCT according to the order by senior ED physician or on-call surgeon who is a member of the trauma team, Informed consent was taken according to the hospital protocol.

All WBCT requested with enhanced single intravenous contrast, requests are excluding non- contrast studies, single organ trauma, and pregnant.
Table 1: The age group of the patients.

\begin{tabular}{|l|l|l|}
\hline Age & Number & Percent \\
\hline $18-30$ & 118 & 50.6 \\
\hline $31-40$ & 49 & 21.0 \\
\hline $41-50$ & 32 & 13.7 \\
\hline $51-60$ & 15 & 6.4 \\
\hline $61-70$ & 6 & 2.6 \\
\hline $71-80$ & 11 & 4.7 \\
\hline $81-90$ & 2 & 0.9 \\
\hline Total & 233 & 100.0 \\
\hline
\end{tabular}

Table 2: The mechanism of trauma.

\begin{tabular}{|l|l|l|}
\hline The mechanism of trauma & Number & Percent \\
\hline MVA & 212 & 91.0 \\
\hline Fall from Hight & 17 & 7.3 \\
\hline Assault & 1 & 0.4 \\
\hline Trauma by heavy object & 2 & 0.9 \\
\hline Gun shot & 1 & 0.4 \\
\hline Total & 233 & 100.0 \\
\hline
\end{tabular}

The information extracted from the hospital database after appropriate ethical permissions, we took the age, sex, mode of trauma, and later the radiology report findings.

\section{Results}

Whole-body CT was carried out on 233 polytrauma patients was arrived at the emergency department (Table 1), 197 (85.5\%) are males 36 (14.5\%) are females, the age of $\mathrm{n} \approx 167(71.6 \%)$ were between $18-40$ yearsold (Table 1 ), $n \approx 212$ (91\%) of those patients mode of trauma is road traffic accidents (RTA), $\mathrm{n} \approx 17(7.3 \%)$ fall from high more the 2 feet, $n \approx 4(1.9 \%)$ were assaulted, trauma by a heavy object and gunshot (Table 2), the outcome of the WBCT were $n \approx 70(30 \%)$ were negative findings, $\mathrm{n} \approx 87(37.3 \%)$ they have a single organ involved in trauma and $n \approx 76(32.7 \%)$ the reports came with multiple organs (more than 1 ) involved in trauma (Figure 1).

The frequency of organs involved in trauma we found chest injuries $n \approx 35(15 \%)$ as an isolated injury head injuries $n \approx 28(12 \%)$, head and chest trauma $n \approx 26$ (11.2\%), while vascular and pelvic injuries are minimal percentages $n \approx 1(0.4 \%)$ (Table 3 ).

In the disposition of the patients, we found that $\mathrm{n}$ $\approx 171(73 \%)$ of the patients were admitted in different wards between intensive care unit and regular wards, and $n \approx 62$ (26.6\%) was discharged from the emergency department.

Further, Bivariate statistical analysis was done with chi-square test, which showed statistical significance with $p$-value $<0.05$ between the number of organs involved in trauma and the positive outcome of WBCT, 


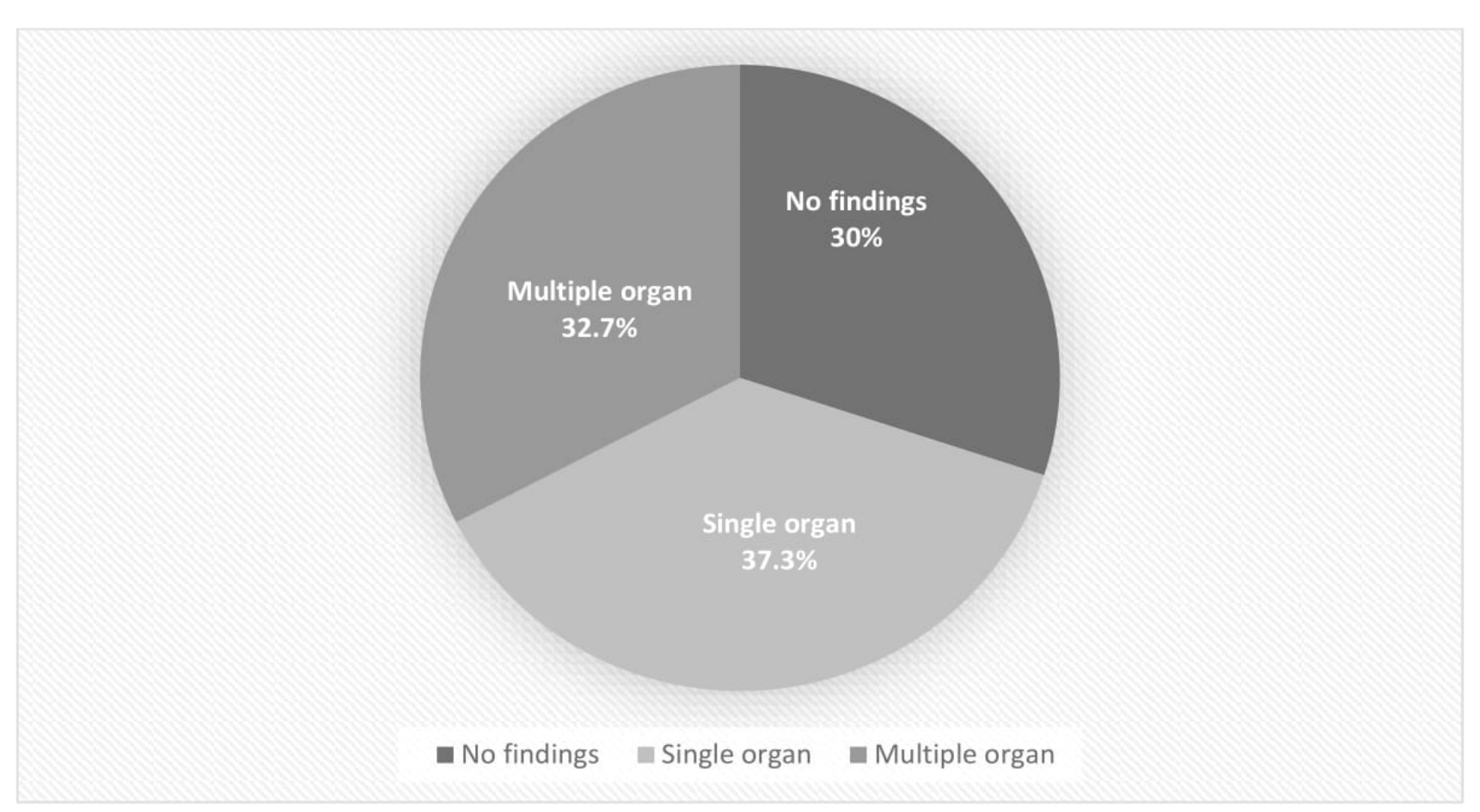

Figure 1: The outcome of WBCT reports.

Table 3: The organs involved in the trauma.

\begin{tabular}{|l|l|l|}
\hline The organ involved in the trauma & Number & Percent \% \\
\hline No organ involved & 70 & 30.0 \\
\hline Head & 28 & 12.0 \\
\hline Neck & 2 & 0.9 \\
\hline Chest & 35 & 15.0 \\
\hline Abdomen & 8 & 3.4 \\
\hline Skeletal & 14 & 6.0 \\
\hline Vascular & 1 & 0.4 \\
\hline Head chest & 26 & 11.2 \\
\hline Head chest abdomen & 11 & 4.7 \\
\hline Head chest abdomen pelvis & 7 & 3.0 \\
\hline Chest abdomen & 2 & 0.9 \\
\hline Abdomen pelvis & 2 & 0.9 \\
\hline Chest abdomen pelvis & 1 & 0.4 \\
\hline Head chest skeletal & 9 & 3.9 \\
\hline Head pelvis & 2 & 0.9 \\
\hline Head abdomen & 1 & 0.4 \\
\hline Abdomen skeletal & 2 & 0.9 \\
\hline Chest skeletal & 2 & 0.9 \\
\hline Head skeletal & 7 & 3.0 \\
\hline Chest pelvis & 1 & 0.4 \\
\hline Head chest pelvis & 2 & 0.9 \\
\hline Total & 233 & 100.0 \\
\hline & & \\
\hline
\end{tabular}

admission of the patients, age of the patient is correlated significantly with the age ( $p$-value $<0.05)$.

\section{Discussion}

There is an increasing concern in health systems regarding the risks of radiation from advanced modalities of computed tomography imaging especially when it is repeated either immediately when patients are transferred between facilities or departments, another concern raises when some articles show that there is a considerable percentage of healthcare workers are not aware of the risks of radiations, which is found around $40 \%$ of emergency departments doctors are not aware of radiation risks in the Australian research, same research done in Kingdome of Saudi Arabia showing the need of more education about radiation risks.

In this study, there is a percentage of $30 \%$ of WBCT done in the emergency department for trauma patients of no findings which are considered in the middle site in percentages in comparison with several international studies which the negative WBCT ranges from $7 \%$ to $57 \%$ in some international centers [8,9], but it is almost the same in comparison with two studies done in KSA which one showing $30 \%$ of negative WBCT in trauma patients and other one fare less around $20 \%$ of negative WBCT [10], this finding in our research although it shows reasonable percentage in KSA but still in the higher limit and raising the concern regarding the unnecessary exposure to radiation which supporting the author's theory, which it might lead to many unfavorable effects for patients from radiation dose although it proved that the chance of causing cancers from medical radiation is very low but also some patients might have their fear and should be considered, also the direct effect is to health economic system and considered part of the unnecessary waste of resources either human or financial resources.

Furthermore, our study found that $37.3 \%$ of the trauma patients had a report of single organ trauma 
mostly head $12 \%$ and chest $15 \%$ trauma respectively, in some international guidelines of trauma management the most first indication for WBCT is trauma to two or more organs, although there is important metaanalysis research showing that WBCT in patients with major trauma will reduce the mortality and the time of admission in the emergency department [11], still, the emergency physicians should assess the needs of WBCT especially when clinically there is one organ from simple trauma and not to use to do WBCT unless for selected patients and senior physicians and surgeons opinions.

We have found that the trauma $91 \%$ of the patients were due to MVA, this might indicate the increasing tendency of emergency physicians or surgeons to request WBCT for trauma patients, appropriate clinical assessment, the use of Focused Assessment Sonography for Trauma (FAST), XRs and local trauma guidelines might reduce this tendency and unnecessary WBCT as well.

Our additional observation during data review and analysis shows that most of the requested WBCT based on the mechanism of injuries, according to international researches in the trauma field the indications of WBCT has multiple criteria including the mechanism of trauma, anatomical criteria, and physiological criteria [12] assisted by others approved international criteria like NEXUS criteria for spinal injuries, NIC criteria for CT brain [13] and FAST scan, along with frequent monitoring combined with the clinical decision [14].

During our review of the database, we noticed some patients who were transferred to $\mathrm{KFH}$ for further management from peripheral hospitals, WBCT have been repeated for them for different reasons like technical issues when they transfer the WBCT data, or for reassessment issues by admitting teams, this observation is out of the scope of this article but we recommend to make further separate statistics and workup regarding this issue to avoid double radiation exposure to the trauma patients within hours or days, also the use of contrast for these patients although Contrast materials are safe; reactions ranging from mild to severe and fatal might happened, severe reactions are uncommon [15]. While serious allergic or other reactions to contrast materials are rare, but the admitting team of the emergency physician should make all the effort to use the same previous WBCT unless there are strong clinical indications and not to be as routine for further documentation.

\section{Limitations}

This study has some limitations. One of the major limitations it is conducted in a single hospital in the region which might limit the results to this facility without integrating the same results from other centers and hospitals in KSA, the study is only done in the patients who are received through the emergency department and missing some trauma patients accepted directly for admission from others hospitals, also we couldn't include the patients who had repeated WBCT in our hospital when it has been done before in others peripheral hospitals due to unavailability of data. finally, all patients who have done WBCT due to trauma are included in the study regardless of their condition on arrival to ED required trauma team activation or not which might indicate the routine utilization of WBCT.

\section{Conclusion}

This study shows a slight overuse of WBCT in our facility, $30 \%$ of reports came of negative findings, and $37.3 \%$ of patients their WBCT reports came with single organ trauma. The combination of senior's clinical decisions; application of appropriate local or international evidence-based criteria for performing WBCT will lead to avoiding a good number of unnecessary $W B C T$, radiation exposure, and side effects of contrast materials besides reducing the costs for the health economic system.

\section{References}

1. Sierink JC, Saltzherr TP, Wirtz MR, Streekstra GJ, Beenen LFM, et al. (2013) Radiation exposure before and after the introduction of a dedicated total-body CT protocol in multitrauma patients. Emergency Radiology 20: 507-512.

2. Ahmadinia K, Benjamin Smucker J, Nash CL, Vallier HA (2012) Radiation exposure has increased in trauma patients over time. J Trauma Acute Care Surg 72: 410-415.

3. Girsa D, Weichet J, Malíková H (2019) Whole-body CT scan and other imaging techniques in examining polytrauma patients-outcomes of a questionnaire survey of trauma centres in the Czech Republic. Acta Chir Orthop Traumatol Cech 86: 334-341.

4. Schafer SB, Rudolph C, Kolodziej M, Mauermann F, Roller FC, et al. (2019) Optimization of whole-body CT examinations of polytrauma patients in comparison with the current diagnostic reference levels. Rofo 191: 1015-1025.

5. Arora R, Arora AJ (2019) Justification of whole-body CT in polytrauma patients, can clinical examination help selecting patients? Quant Imaging Med Surg 9: 636-641.

6. Jawad H, Raptis C, Mintz A, Schuerer D, Mellnick V (2018) Single-contrast CT for detecting bowel injuries in penetrating abdominopelvic trauma. AJR Am J Roentgenol 210: 761-765.

7. Andreucci M, Solomon R, Tasanarong A (2014) Side effects of radiographic contrast media: Pathogenesis, risk factors, and prevention. Biomed Res Int 2014: 741018.

8. Oosthuizen GV, Bruce JL, Bekker W, Shangase N, Laing GL, et al. (2016) Pan computed tomography for blunt polytrauma: Are we doing too many? S Afr Med J 106: 801-803.

9. Linder F, Mani K, Juhlin C, Eklöf H (2016) Routine wholebody CT of high energy trauma patients leads to excessive radiation exposure. Scand J Trauma Resusc Emerg Med 24: 7.

10. Maghraby $\mathrm{NH}$, Alshaqaq HM, Al Qattan AS, Alfaraj AF, Alghamdi OA, et al. (2020) Negative whole-body computed tomography scans in polytrauma patients: A retrospective cohort study. Open Access Emerg Med 12: 305-313. 
11. Jiang L, Ma Y, Jiang S, Ye L, Zheng Z, et al. (2014) Comparison of whole-body computed tomography vs selective radiological imaging on outcomes in major trauma patients: A meta-analysis. Scand J Trauma Resusc Emerg Med 22: 54

12. Al Orf A, Waheed KB, Baig AA, Mohammad KS, El Sirafy $\mathrm{MN}$, et al. (2018) Patterns of injury detected by pancomputed tomography after road traffic accidents: A retrospective review from a trauma center in Saudi Arabia. Ann Saudi Med 38: 245-250.

13. Shravat BP, Huseyin TS, Hynes KA (2006) NICE guideline for the management of head injury: An audit demonstrating its impact on a district general hospital, with a cost analysis for England and Wales. Emerg Med J 23: 109-113.

14. Asha S, Curtis KA, Grant N, Taylor C, Lo S, et al. (2012) Comparison of radiation exposure of trauma patients from diagnostic radiology procedures before and after the introduction of a pan scan protocol. Emerg Med Australas 24: $43-51$

15. Kobayashi D, Takahashi O, Ueda T, Deshpande GA, Hiroko A, et al. (2013) Risk factors for adverse reactions from contrast agents for computed tomography. BMC Med Inform Decis Mak 13: 18. 